\title{
De la exclusión a la necesidad de una transformación de las miradas sobre la discapacidad intelectual: análisis enfocado en la cuestión del Síndrome de Down
}

\author{
From exclusión to the need for a transformation of views on intelectual disability: \\ analysis focused on the issue of down syndrome
}

* Edward Herrera Sanclemente

**Tania Collazos Rodríguez

\section{Resumen}

El presente documento tiene dos objetivos fundamentales: en primera instancia, analizar algunos desarrollos conceptuales sobre los cambios presentados en el abordaje de la discapacidad intelectual, haciendo énfasis en el síndrome de Down. Para esto, se propone iniciar la pesquisa con elaboraciones teóricas y conceptuales sobre el concepto de síndrome de Down $\mathrm{y}$, posteriormente, se exponen hechos que evidencian cambios tanto en el panorama legislativo, como en la comprensión del fenómeno. En segunda instancia, se analiza el modo en que emerge la necesidad de articular el discurso legislativo con verdaderas garantías materiales que faciliten el desarrollo de la individualidad. Al mismo tiempo, es necesaria la promoción de un cambio de mirada en la sociedad que incorpore valores y perspectivas que tomen distancia del determinismo de la enfermedad. La educación, en este sentido, se convierte en un escenario clave para llevar a cabo transformaciones que involucren no solo cuestiones del orden académico, sino también asuntos del orden personal y afectivo, tanto a nivel individual como en otros escenarios, por ejemplo, la familia.

Palabras clave: Inclusión, educación, síndrome de Down, enfermedad, discapacidad, aprendizaje, autonomía.

\section{Abstract}

This paper has two main objectives: the first one is to analyze some theoretical developments made according to the changes in the understanding of intellectual disability, putting emphasis in Down Syndrome. For this, the purpose is start the research with conceptual elaborations on Down Syndrome and expose some facts that show adjustments as much in the

* Psicólogo, Universidad del Valle. Magíster en Filosofía, Universidad del Valle. Candidato a doctor en Filosofía, Universidad del Valle. Investigador Grupo Lumen Humanitas. Fundación Universitaria Católica Lumen Gentium. eherreras@unicatolica.edu.co

** Psicóloga, Universidad del Valle. Magíster en Psicología, Universidad de San Buenaventura Cali. Investigador Grupo Lumen Humanitas. Fundación Universitaria Católica Lumen Gentium. tcollazos@unicatolica.edu.co 
legislative context as the grasp of this phenomenon. Secondly, we examine the arise of the need to establish a link between the legal discourse and real material guaranties of growth of individuality. At the same time, the promotion of a change in the societal view is needed through the incorporation of values and perspectives that take distance from the illness determinism. In this sense, education turns into a key to carry out shifts, not only in an academic way, but also in personal and affective scenarios such as the family.

Keywords: Inclusion, education, Down Syndrome, sickness, disability, learning, autonomy

\section{Generalidades del Síndrome de Down y su relación con la inclusión- exclusión social}

Los conceptos elaborados para pensar el síndrome de Down muestran una nosología de abordaje que lleva a clasificar y comprender, tanto causas, como evidencias que rodean esta condición. El hecho de crear un campo de entendimiento para dicho fenómeno, facilita una mirada que involucra una transformación de la realidad de las personas con síndrome de Down. En este sentido, podría señalarse la propuesta inicial descrita por John Langdon Down en 1866 (citado por Stephanie Lorenz, 1998), cuyos acercamientos al síndrome de Down estaban orientados a clasificar individuos entre grupos étnicos, usando características como su tipo de cabello, la estructura ósea y el color de piel. Propuso, además, que los llamados, en aquel entonces, débiles mentales, deberían ser clasificados en tipos raciales tales como etíope, azteca, malayo o mongol. Se presentaba pues, una terminología para tratar de entender una particularidad presentada en un grupo de personas. Sin embargo, los desarrollos posteriores comenzarían a trabajar a partir de las dificultades en el desarrollo físico y mental y, por ende, la cuestión se orientó paulatinamente a facilitar una comprensión diversa de esta condición genética.
Respecto a conceptos más recientes, Brill (2006) plantea que el síndrome de Down ${ }^{1}$, es una condición que transforma el desarrollo mental y físico; condición que presenta como características fenotípicas tener dedos cortos o palmas notablemente arrugadas. Además, esta perspectiva, señala la existencia de dificultades para el desarrollo intelectual, lo cual implica que el aprendizaje de estas personas estará condicionado a unos procesos más lentos que el promedio de quienes que no están dentro de esta condición. Complementando esto, para definir el síndrome de Down también se usa la expresión "retraso del desarrollo" según lo planteado por Bowman-Kruhm (2003, p. 18), mostrando una visión que compromete las capacidades que son inherentes a los procesos vitales en el ser humano. Por ejemplo, los niños en promedio desarrollan aprendizajes como caminar, hablar, usar herramientas, pero en el caso de los niños con SD estas destrezas aparecen en otro momento posterior al hito de desarrollo típico.

En este caso también se implican las condiciones biológicas determinantes que interceden en los procesos de desarrollo. En concordancia con esto, para Forrest Keel (2016), el SD es también es una condición genética, donde el factor determinante se define por la aparición de un cromosoma extra en la biología del individuo. En términos generales, las personas tienen 46 cromosomas, pero en el caso de alguien con SD su herencia genética determina 47, encontrándose así una explicación desde la ciencia. Lorenz (1998) menciona que esto se debe a una falla en el proceso de división celular, razón por la que se presenta una copia del cromosoma 21.

Además de esto, se han elaborado unas claves para establecer definiciones a partir de lo encontrado en la práctica clínica. Por ejemplo, para el diagnóstico, Reynolds, Zupanick y Dombeck (s.f.), exponen unos criterios que abarcan desde el uso del razonamiento, pasando por la resolución de problemas, planeación, pensamiento abstracto, juicio, aprendizaje académico y la experiencia de

1 A partir de este punto y durante el desarrollo del texto se utilizará la sigla SD para hacer referencia al Síndrome de Down. 
aprendizaje. Sobre estos dos últimos, el aprendizaje académico se entiende como el contexto en el que se desarrolla la habilidad de aprender mediante el uso de métodos tradicionales. En el caso del segundo, se entiende como la habilidad para aprender a través de la experiencia, la observación y el ensayo-error. Estas habilidades son evaluadas mediante test de IQ.

No obstante, a pesar que ha habido un cambio en la conceptualización del SD desde que la propuso John L. Down, el asunto de la discriminación ha estado presente de algún modo en la comprensión y en la práctica. Por ejemplo, Mc Cabe y Mc Cabe (2001) afirman que se ha presentado una exclusión desde el campo genético con las personas que presentan alguna discapacidad intelectual y esto puede evidenciarse en el desarrollo de saberes influenciados por la eugenesia. En Estados Unidos, por ejemplo, con la aparición de personajes como Foster Kennedy en las primeras décadas del siglo XX (Noak y Fangerau, 2004), se hacía explicita una mirada que abogaba por una exclusión y eliminación de los individuos dado que se les consideraba un problema de salud a nivel de la población general. En tal sentido, no deben subestimarse los datos históricos donde predomina el modelo eugenésico de la discapacidad, caracterizado por la deshumanización (estar por debajo del estado de humanidad o, por el contrario, por encima de ella, como es el caso de considerarles seres con características místicas, angelicales o sagradas), donde se incluían a las personas con SD.

Desde este punto de vista, sería entonces interesante citar brevemente algunos antecedentes de estas ideas. En la época prehistórica, por ejemplo, las personas con discapacidad eran objeto de eliminación por la imposibilidad de llevar una vida independiente y no poder contribuir al crecimiento del grupo social (Naciones Unidas, 1956, Citado por Gómez, 2005). Igualmente, en épocas antiguas, se afirma que Platón hablaba de los caricatos, quienes estaban relacionados con seres mitológicos cuya función era comunicar mensajes provenientes de los dioses (Inzúa, 2001).
Ya en tiempos recientes, hablando particularmente de la modernidad, aparecen estrategias de abordaje de la enfermedad cuyas denominaciones como la de feebleminded (débil mental), o enfermo, hacían y continúan siendo parte del repertorio de etiquetas médicas, fiel reflejo de un modelo eugenésico de la discapacidad. Para ejemplificar esto, sería necesario referenciar la alusión hecha en el Congreso de la Federación Internacional de Organizaciones Eugenésicas en los años 20 (siglo XX) que promulgaba una urgencia para familiarizar a los países civilizados con los problemas de la herencia genética y los estudios sobre poblaciones y eugenesia (Kühl, 1994). Otro ejemplo más específico está en la incorporación de estas ideas por países como Alemania, el cual, durante el periodo comprendido entre los años 1933 y 1945, consideró a las personas discapacitadas mentales como objeto de esterilización involuntaria y eliminación (González, 2010).

Se buscaba entonces una solución desde el campo del saber científico para prevenir una posible degeneración de la raza humana, por lo que era necesario impedir que las personas con SD contrajeran matrimonios o que tuvieran hijos entre sí. Esta visión, de alguna manera, tendrá un correlato en tiempos recientes y hay datos que merecen una reflexión como es el caso del descenso de las tasas de nacimientos de niños con SD en Asia y Europa. Regiones como Irán presenta un porcentaje de $0,3 \mathrm{~b} \%$ de nacimientos y España un 0,6 por mil, esto, seguramente, debido a la legalización del aborto electivo (Nazer y Cifuentes, 2011). También surge interés por el caso del Reino Unido, donde un 90 \% de las personas que saben que su hijo nacerá con SD hacen uso del aborto (BBC, 2016). En concordancia con esto, Islandia es considerado el primer país sin niños con SD, puesto que interrumpe el $100 \%$ de los embarazos que presentan dicho diagnóstico (Bell, 2017). Esta información, si bien no pretende derivar en generalizaciones, muestra que a pesar de los cambios en las denominaciones sobre el SD 
y la discapacidad intelectual, hay una cuestión que busca prevenirla, en tanto no hace parte de las condiciones normales de vida.

En este punto, es importante señalar la referencia que hace Palacios y Romañach (2008), acerca de la transformación de las formas de comprender lo que llama la Diversidad Funcional (Palacios y Romañach, 2008) bajo tres perspectivas: los modelos de Prescindencia, Rehabilitador y Social. En el primer modelo, prescindencia, las causas son atribuidas a relatos religiosos o castigos divinos, por tanto, las personas nacen con la condición de discapacidad por algún tipo de influencia diabólica o porque sus vidas no merecen la pena ser vividas, dado que son producto de algún tipo de castigo de los dioses. El segundo modelo, llamado rehabilitador, plantea que los origines de la discapacidad no son religiosos sino científicos, por tanto, a través del saber de la ciencia, se busca la normalización de las personas, valorando el éxito de las intervenciones por la cantidad de destrezas y aprendizajes logrados por los individuos mediante procesos sistemáticos. Y finalmente, en cuanto al tercer modelo denominado social, los autores afirman que refiere a un planteamiento en el que las causas de la discapacidad no son científicas sino sociales. Es decir, que deben encontrarse en las discusiones sobre los derechos humanos y el reconocimiento de valores como la dignidad, igualdad y libertad individual de las personas, las claves para superar las barreras de la discriminación (Palacios y Romañach, 2008).

Para concluir aquí, las cuestiones ligadas a la reproducción de una raza sin alteraciones o enfermedades, cambiará con la aparición de políticas encaminadas a promover la igualdad de derechos, presentando retos tanto a las instituciones sociales como a las políticas, en tanto a la educación o atención que deben brindar a la población con alguna discapacidad intelectual, lo cual refleja un cambio de comprensión de la discapacidad, tal y como se presenta en la aparición de modelos como el social (Palacios y Romañach, 2008).
Durante el desarrollo de las concepciones sobre discapacidad y del SD, emergen unas transformaciones que invitan a ampliar los puntos de vista, pues en sí mismos los modos bajo los cuáles son explicados aspectos de la discapacidad reflejan posiciones determinantes. Estos, por tanto, forjan límites que más allá de representar posturas teóricas o incluso históricas, impiden la materialización de luchas por el reconocimiento de las capacidades individuales que potencialmente pueden desarrollar las personas con SD si hubiera otro punto de referencia para su comprensión. Por ello, vale la pena discutir sobre algunos aspectos puntuales por medio de los cuáles se pretende brindar unas pautas para el análisis. En tal sentido, se traerá al debate cuestiones como la educación y la necesidad de pensar en modos de independencia o autonomía.

\section{La educación y las posibilidades de socialización en personas con Síndrome de Down: una búsque- da hacia la superación de barreras por medio de la materialización de garantías.}

La educación, como mecanismo de socialización, tiene varios fines entre los que se encuentra procurar que cada persona sea sensible a las necesidades de los demás individuos, inculcando valores como la solidaridad dentro del ejercicio de la libertad (Hurtado y Agudelo, 2014). Representa también un factor determinante para construir una perspectiva socio económica, desde el punto de vista del desarrollo nacional, dado que provee de las herramientas para que los ciudadanos gestionen sus potenciales en un contexto laboral. Además, sobre este asunto hay datos empíricos que evidencian que las personas con discapacidad intelectual poseen niveles más bajos de educación, ingresos y hábitos financieros como el ahorro en paralelo a la población general (Hernández y Hernández, 2005). Según el reciente informe de la Organización de las Naciones Unidas ONU (2016), acerca de las necesidades más sentidas para 
la población con discapacidad en Colombia, entre las que se encuentran las de carácter intelectual, se encuentran aquellas relacionadas directamente con la educación ya que notifican que actualmente se presentan bajos niveles de participación de esta población en todos los niveles educativos. Además, el rechazo y discriminación es frecuente en las aulas regulares, resaltando así la falta de formación en pedagogía de quienes tienen a cargo estos grupos en escuelas regulares, predominando las llamadas aulas especializadas, donde no es del todo clara la propuesta educativa para lograr aprendizajes significativos.

De esta forma, se evidencia una exclusión explícita hacia este grupo de personas, cuya brecha puede disminuirse a través de las herramientas que brinda la educación. No obstante, es en el campo de los derechos humanos que comienza a vislumbrarse un cambio. Por ejemplo, con La Convención de los Derechos de las Personas con Discapacidad que entró en vigencia en diciembre de 2006, hubo un foco de atención con miras a atacar la prevalencia de prácticas discriminatorias de la sociedad con este grupo de personas. Se encuentra, pues, pasajes como el artículo 23 que plantea que las personas con discapacidad disfrutarán de igualdad de oportunidades y tendrán acceso a la educación. De igual modo, en el artículo 24, se afirma que los Estados deben asegurar su acceso a los niveles de primaria, secundaria y profesional, promoviendo además la participación en la sociedad, el desarrollo del potencial personal, sus talentos y creatividad. Sin embargo, la implementación de estos derechos en el Estado colombiano, deja más motivos de preocupación, que de reales logros significativos (ONU, 2016).

A pesar de esto, en cuanto a las normatividades precedentes, se encuentran aquellas que refuerzan estas ideas basadas en los derechos que una persona con discapacidad debería tener garantizados; La Declaración Mundial Sobre Educación para Todos de 1990, por ejemplo, plantea la necesidad de la universalización del acceso a la educación y la promoción de la equidad. Asimismo, está la
Declaración deSalamanca como un marco de acción para las necesidades educativas, la cual, además, tiene como principio rector que las escuelas "deben acoger a todos los niños, independientemente de sus condiciones físicas, intelectuales, sociales, emocionales, lingüísticas u otras" (Declaración de Salamanca, 1990, p. 6) dando acceso a niños en situación de discapacidad, aquellos en condiciones de calle y niños de grupos desfavorecidos.

Este marco legal muestra un contexto de interés por el estudio de la inclusión y la discapacidad intelectual en el contexto de la educación, al mismo tiempo que permite evidenciar la transformación de la mirada sobre las personas con discapacidad cognitiva en el ámbito educativo. Por lo tanto, los países que se adscriben a estas normas o tratados deben generar las condiciones para que esto se materialice en propuestas de trabajo con la población, respondiendo a los diagnósticos de algún tipo de situación excluyente.

En esta vía existen iniciativas que llevan a pensar en la materialización de políticas de inclusión. Por ejemplo, Yeepay y Chwen (2012) indagaron sobre la participación social y comunitaria de adolescentes con SD en Taiwán, tratando de encontrar diferencias en relación con la edad, género y las funciones cognitivo-motoras. En sus conclusiones, consideran que los jóvenes con este tipo de síndrome participan en actividades formales presentando un grado de compromiso de su parte, pero posteriormente, a medida que envejecen, esto se presenta en menor medida. Probablemente esto se deba al cambio de dinámicas sociales a lo largo de la vida de las personas (Yeepay y Chwen, 2012).

De acuerdo con este contexto, la participación presenta limitaciones porque las situaciones de exclusión aparecen con mayor frecuencia, generando así menor intercambio social. En consecuencia, el acceso a espacios de práctica deportiva o relacionados con el ejercicio corporal disminuye $y$, por ende, las personas entran en un modo de vida sedentario conllevando a futuros problemas de 
salud (Yeepay y Chwen, 2012). En Colombia, esta cuestión ha sido poco documentada, sin embargo, existe una legislación que introduce la filosofía de la inclusión de las personas con SD desde la categoría general de discapacidad.

De este modo, por ejemplo, la Ley 1618 de 2013, en el artículo 18 (sobre el Derecho a la Recreación y el Deporte), afirma que el Estado colombiano es un garante del derecho a participar en actividades culturales, recreativas y deportivas de las personas con discapacidad. Además, contribuye a fortalecer el sistema competitivo paralímpico y fomenta la práctica del Deporte Social Comunitario, siendo este un proceso de inclusión que conlleva a potenciar los aprendizajes y habilidades de las personas con discapacidad en función de su ciclo vital (República de Colombia, Ley 1818 de 2013).

A pesar que experiencias como las de Yeepay y Chwen (2012) muestran una realidad que está fuera del contexto de interés y en la medida que las dinámicas socio culturales son diversas, el aporte de este documento está encaminado a reflexionar sobre las necesidades latentes de la población con síndrome de Down a lo largo del proceso vital y, al mismo tiempo, de la diversidad de posibilidades de trabajo que puede ofrecerse en esta vía. Por lo tanto, es de notable interés la labor de evidenciar las diferencias individuales que se relacionan con los intereses, motivaciones y capacidades de aprendizaje, así como también la promoción de una diversidad de experiencias de socialización y conocimiento (Valls Gubern, 2010). En concordancia con esto, se hace necesario que terapeutas, profesores y cuidadores faciliten espacios para desarrollar actividades de socialización y así poder aumentar las experiencias de aprendizaje en las personas con SD (Yeepay y Chwen, 2012).

Según lo dicho por Valls Gubern (2010), hablando específicamente del tema educativo, en la escuela el propósito es promover la formación integral contribuyendo a alcanzar el máximo potencial de la persona en los planos personal y social.
Asimismo, plantea que la escuela, como institución que ha sufrido transformaciones, permite incluir a todos los estudiantes independientemente de sus características individuales. Dicha cuestión busca además revaluar el lugar tanto del docente como del estudiante, por tanto, el docente ya no es la persona que posee la información sino quien ejerce un acompañamiento al proceso de aprendizaje. El profesor, pues, facilita un entorno de socialización buscando el reconocimiento y auto conocimiento de la discapacidad como aspectos necesarios para el crecimiento y maduración de quienes aprenden (Valls Gubern, 2010). El hecho de poder descubrirse a sí mismo, hace posible la elaboración de competencias sociales para interactuar con otros y desarrollarse como persona, por tanto, niños y adolescentes que puedan adquirir este saber, podrán afrontar los retos de sus entornos en la medida que son aceptados como personas y se posicionan como protagonistas de sus acciones (Valls Gubern, 2010).

De acuerdo con Garvía y Ruf (2014), la integración de las personas con SD en espacios sociales, tales como escuelas y lugares de trabajo, ha facilitado pensar en cuestiones como la independencia y en la posibilidad de tener un hogar. La emancipación de sus propias familias es entonces una cuestión excepcional, pero supone un acontecimiento que muestra la escogencia de un proyecto de vida elegido de manera voluntaria (Garvía y Ruf, 2014). De esta forma, si se lograse esto, se tendría un contexto que facilita cuestiones como el auto reconocimiento y, por ende, también la inclusión de las personas en escenarios que hasta cierto punto estaban pensados solo para personas que no hacen parte de la condición del SD.

Esto, además,implica la construcción deidentidad que, según Valls Gubern (2010), está mediada por la posibilidad de tener un rol activo en los diversos escenarios en los que puede participar una persona con SD. Así las cosas, al ingresar en un grupo social, cultural, deportivo o del mundo laboral, la persona puede sentirse valorada y comprendida por lo que es; alguien en proceso de crecimiento y con miras a 
lograr otros estados de desarrollo. En este sentido, los grupos sociales como la familia y contextos como la escuela, facilitan las herramientas necesarias para incluir a las personas en otros ámbitos sociales y brindar una autonomía que podría extenderse más allá de los espacios educativos o institucionales, llegando incluso a pensar en el manejo de un mayor nivel de independencia. Por tanto, la tarea de las instituciones radica en la promoción de un desarrollo psicoafectivo que involucre el aprendizaje de habilidades sociales y acompañamiento en el proceso tanto de integración a la sociedad como de formación de pensamiento.

El desarrollo de las capacidades para lograr una autonomía en las personas con SD, por una parte, contribuye a superar posiciones como la excesiva dependencia e infantilización por parte de los adultos cuidadores y por otra, a que se releve al Estado gran parte de su responsabilidad en ofrecer las garantes de educación para una población que desea tener más participación en el entramado social. Es decir, que aquellas formas de crianza en las que los hijos son tratados como si fuesen niños, aun cuando no lo son, si bien son promovidas desde un imaginario de lo correcto, no fomentan a largo plazo un desarrollo que contribuya a dar un lugar distinto a los hijos y, además, no permiten que se asuman otros roles que llevarían a lograr una autonomía en las personas con SD tal y como lo plantean Garvía y Ruf (2014).

Es necesario, pues, que el trabajo de la educación se presente de forma multidireccional, cuya tarea esencial no recaiga solo en las familias, ya que la falta de autonomía puede ser la consecuencia de sus acciones directas, pero también de un Estado que poco reconoce sus necesidades reales. En consecuencia, surge la sobreprotección como mecanismo que, si bien preserva a la persona con $\mathrm{SD}$ de una sociedad que es percibida como poco comprensiva e incluyente, termina marginándola y cerrándola a los grupos de socialización primaria, como es la familia misma, o a instituciones donde solo comparten con personas de su misma condición, por ejemplo, escuelas y aulas especiales.
Esta consecución deautonomía, podría evidenciar la capacidad de cada persona con SD para construir relaciones que no impliquen una total dependencia. El ámbito de las relaciones de pareja, por ejemplo, ayuda a pensar esta cuestión, sobre todo en el tema de la sexualidad. Según Garvía y Ruf (2014), la sexualidad en el escenario de la discapacidad es un acto que va más allá del coito, por ende, permite establecer un mejor vínculo con cuestiones como la atracción, el deseo y la posibilidad de brindar estabilidad en una relación de pareja. A pesar de esto, las personas con SD poseen una limitación en dicho sentido, puesto que, al tener una carencia de apoyo a nivel social y familiar para emprender un proyecto de vida en pareja, surgen impedimentos que no permiten el desarrollo de esta cuestión. Así, cuando las personas con SD establecen relaciones afectivas con personas de igual condición, sus progenitores inciden notablemente en la dinámica y el desarrollo de estas, condicionando muestras de afecto y prácticas propias de una relación en la que podrían llegar hasta el acto sexual mismo (B. Garvía y P. Ruf, 2014).

Seguramente, algunas razones por las cuáles las familias intervienen en las relaciones afectivas de sus hijos, es porque podrían percibirlos como niños a pesar que ya han superado la infancia en muchos casos, o como personas con poca autonomía para asumir una relación de pareja, más allá de las idealizaciones románticas. Si bien podría pensarse que los padres intervienen de esta manera por algún tipo de actitud sobreprotectora o capricho personal, habría que ampliar el análisis y comprender que las condiciones educativas y socio-económicas no garantizan oportunidades para que los jóvenes puedan madurar en términos académicos y productivos. Por lo tanto, los padres de familia intervienen directamente en la crianza de sus hijos y en todos sus asuntos, incluso aquellos que son de índole personal como los noviazgos o las relaciones sexuales, dado que si llegase a ocurrir un embarazo producto de estos vínculos, serían los mismos padres quienes tendrían que asumir la responsabilidad del caso. 
La autonomía y el ejercicio del rol de padre están estrechamente relacionados en este caso, pues el sostenimiento económico, la crianza de un hijo y las decisiones importantes para la vida, constituyen aspectos interdependientes. A pesar de esto, el modelo de desarrollo de la sociedad basado en la productividad, implica hacer uso de competencias de escritura, lectura y habilidades matemáticas para poder encontrar oportunidades laborales. Entonces, la educación y el desarrollo vocacional en los jóvenes se presentan como una necesidad que debe ser atendida desde temprana edad y de forma permanente durante la adultez, para alcanzar un estado de mayor independencia.

En relación con esto, existen puntos de vista que reflejan incredulidad al pensar que una persona con SD podría sostener una familia o, incluso, que sean capaces de transmitir conocimientos indispensables para la supervivencia de generaciones posteriores. Además, dado el hecho que hay conceptualizaciones sobre el tema que sustentan que las personas con SD presentan un desarrollo intelectual y motor diverso en comparación a quienes no han tenido esta condición (Bowman-Kruhm, 2003), entonces tales diferencias son pensadas bajo la óptica de unos determinantes.

No obstante, reflexiones como las de Azevedo y Damasceno (2013), brindan elementos de tipo empírico que permiten pensar en otras posibilidades de desarrollo en la cuestión de la sexualidad y la familia. Estos autores presentan un caso que en la actualidad es de carácter escaso y excepcional, en el que una mujer con SD contrae matrimonio $\mathrm{y}$, además, tiene una pareja de su misma condición con quien tiene un hijo que nace sin SD. En consecuencia, se plantea un dato interesante que pone en cuestión las creencias sobre la imposibilidad de construir una noción de familia dentro de la discapacidad cognitiva $\mathrm{o}$, incluso, de poder pensar una sexualidad bajo esta condición. De hecho, se conoce que las ideas personales sobre la sexualidad son influenciadas por las generalidades que se establecen en este tema a nivel cultural. La liberación sexual en la sociedad, si bien facilita una amplitud de información acerca de este tema, también genera que en algunos casos exista una banalización de la misma y, por tanto, esta cuestión también es recibida en el contexto de quienes viven con discapacidad intelectual y acceden a este tema. En palabras de los autores referenciados anteriormente: "ellos también quieren construir una imagen de normalidad en lo afectivo y de competencia sexual" (Azevedo y Damasceno, 2013. p. 16). Es decir, se plantea una necesidad de pensar la sexualidad más allá de los niveles culturales, dado que estos contribuyen a generar creencias o, incluso, prejuicios que constituyen un obstáculo para pensar en otras formas de desarrollo para los individuos con SD. Vale la pena aclarar que este caso excepcional, refleja un modo de pensar las relaciones afectivas en personas con SD, cuyo escenario social de fondo es particular.

Si bien, esta referencia muestra una posibilidad por medio de la cual las personas pueden lograr alguna forma de desarrollo, es pertinente señalar una correspondencia con las condiciones materiales del contexto social y político que garantiza, a través de mecanismos institucionales, otro tipo de relaciones y dinámicas favorables. Para este caso, Brasil es el país que enmarca tal experiencia y donde las leyes proporcionan los medios para garantizar más que el reconocimiento de la norma, un acceso igualitario y material conllevando a la inclusión social (Hernández, 2017).

Para Crosso (2010), por ejemplo, en Brasil ha habido un financiamiento favorable desde entidades como el Fondo de Desarrollo de la Educación Básica (FUNDEB), encargada de dar financiamiento para la educación que destina un $20 \%$ para las personas con discapacidad. Además, acoge la resolución No 13 del Consejo Nacional de Educación que establece que el FUNDEB financia la permanencia de un estudiante tanto en un centro de educación especial como en una escuela regular. 
Es decir, que esta regulación no hace hincapié en que la educación especial deba reemplazar la formal, sino que ambas deben complementarse. Entonces, el abordaje de la discapacidad intelectual y el SD implica, además, la participación social, levantamiento de prejuicios y el acceso a derechos que permitan garantías desde la primera infancia, facilitando el acceso al entramado social en distintos niveles. Seguramente es en esta vía que puede pensarse en la generación de espacios para las libertades individuales, tales como el desarrollo psicoafectivo y sexual. En otras palabras, requiere de trascender las fronteras del derecho plasmado en el papel, para hacerlo visible en las calles y espacios cotidianos.

Podría sintetizarse, entonces, que de lo dicho anteriormente surge una necesidad de cambio a nivel cultural que no solo encuadre en lo educativo, sino que también permita superar estrategias basadas en brindar información sobre la discapacidad intelectual o que busca categorizar los tipos de comportamientos presentados en el SD formulando determinantes. Se requiere además de la eliminación de estereotipos como aquellos que promueven que la persona con SD no puede regularse a sí misma, por una visión que privilegie un enfoque crítico de los mecanismos que permiten la materialización de los derechos de estas personas, superando barreras tanto en la escuela, como en diferentes espacios donde aún perdura una mirada excluyente. En esta mirada, la sociedad debe transformarse reconociendo las diferencias que la componen, al igual que es menester la articulación de la política pública con las dinámicas de la sociedad, de modo que los derechos sean reconocidos ampliando el horizonte de dignidad humana que conlleve así a un equilibrio en la calidad de vida de las personas (Maldonado, 2013).

De igual modo, pensando en cuestiones individuales como la sexualidad, hay que educar en una idea que vaya más allá de un acto reducido al coito. Seguramente la noción de autonomía se encuentra subyacente a este asunto, en la medida que si existe una auto regulación del propio cuerpo, podría haber una regulación en otros asuntos del desarrollo vital, incluyendo el proyecto de vida hasta el momento de la vejez.

Sobre este asunto particular, es pertinente señalar lo dicho por Esteba-Castillo, Ribas, Baró y Novell(2006), quienesseñalanqueelenvejecimiento es otra cuestión necesaria al pensar el tema de la discapacidad intelectual. Según lo dicho aquí, la expectativa de vida de las personas con SD se encuentra en un $80 \%$ en personas que tienen una edad aproximada de 50 años, llegando en algunos casos hasta los 60 y 70 años (Esteba-Castillo, Ribas, Baró y Novell, 2006). En consecuencia, con miras a promover un lugar distinto de estas personas en la sociedad, deben considerarse algunas premisas en tanto que la sola discapacidad sería objeto de exclusión, por lo cual, el envejecimiento sería una cuestión que profundizaría el asunto.

\section{El papel de los cuidadores y educadores en la labor inclusiva}

Otro asunto importante que plantea la transformación de la mirada sobre la discapacidad intelectual, es el papel del cuidador. Por las características mismas del tipo de discapacidad en el SD, las personas generalmente cuentan con el apoyo de un familiar que hace el rol de acompañante, en tanto facilita los cuidados necesarios de salud, pero también contribuye a la garantía del reconocimiento de la persona como alguien que posee derechos. Por esta razón, es pertinente pensar en la importancia de su papel como receptor de la carga de la condición de discapacidad del otro a quien cuida. Según Esteba-Castillo et al., (2006), en los cuidadores es común encontrar una sobrecarga en el sentido emocional y físico.

Esto sucede, sobre todo, cuando las personas con síndrome de Down padecen, además de su 
discapacidad intelectual, alguna enfermedad que acentúa su condición, por ejemplo, Alzheimer o algún tipo de demencia. En un estudio realizado con 285 participantes con una edad promedio de 46.5 años, la prevalencia de demencia tipo Alzheimer se encontraba en un $13.3 \%$, lo que muestra un escenario posible que debe ser abordado como factor de garantía de bienestar para las personas con SD (Esteba-Castillo et al., 2006). En tal sentido, algunas medidas tomadas para reducir la carga del cuidador pasan por medidas psicoeducativas, apoyo emocional y técnicas de apoyo por línea telefónica. En términos generales, el cuidado de una persona con SD se convierte en una carga emocional muy alta que deben soportar las familias y, más aún, si la persona además del SD posee otra enfermedad como la demencia.

Por lo tanto, debe existir una estrategia que permita abarcar las necesidades tanto de los pacientes como de sus cuidadores (Esteba-Castillo, et al., 2006). Pero a pesar de esto, las familias pueden transformar su experiencia de cuidado de ser asumida como una carga, a un proceso de vida en el que hallan satisfacciones como el avance de sus hijos con SD en la escuela, logros deportivos o de actividades que adquieren un significado diferente a una condición de enfermedad. La crianza en este sentido es re valorada y aceptada, no en función de un cuidado de un enfermo, sino de un sujeto con el que se desarrolla un vínculo (Collazos y Herrera, 2017).

Ahora bien, al trasladar esta cuestión en países de Latinoamérica, por ejemplo, Colombia, se encuentra que con la Constitución de 1991 se elabora un nuevo modo de pensar la educación. Debido a la receptividad hacia las políticas internacionales en Colombia, se construye un nuevo marco legal para trabajar en beneficio de las minorías en este contexto, pues las cifras de personas con alguna condición de discapacidad adquieren una relevancia en términos estadísticos para el gobierno. Así, en el año 2005 según el Departamento Administrativo Nacional de Estadística DANE (citado por Córdoba,
Mora, Bedoya y Verdugo, 2007), el censo general de la población con discapacidad en Colombia arrojaba la cifra de 2.640 .000 personas; y, en cuanto a la prevalencia de la discapacidad, existen datos que contribuyen a precisar el diagnóstico, entre estos, que la discapacidad aumenta con la edad, teniendo una mayor incidencia a partir de los 45 años. También se encuentra que las principales causas están asociadas a enfermedades y la violencia, así como también que el nivel educativo de personas y hogares con discapacidad es más bajo en comparación con la población que no tiene condición alguna de incapacidad. Además, otros datos interesantes, plantean que la participación social de las personas con discapacidad es baja y que las familias que tienen uno de sus integrantes con alguna condición de inhabilidad son más vulnerables económicamente (Plan Indicativo de Discapacidad, 2005, citado por Córdoba et al., 2007).

Otros acercamientos a la cuestión como el de Padilla (2011), sostienen que según los datos del DANE del censo del año 2005, a esta fecha había 400313 menores de 18 años en situación de discapacidad. De esta cifra, un $9.1 \%$ tenía discapacidad motriz, $19.8 \%$ discapacidad mental, $14 \%$ discapacidad sensorial y $34.8 \%$ discapacidad cognitiva. Además, también plantea que, según los datos de esta fuente nacional, el $22.5 \%$ de las personas con discapacidad eran analfabetas para el año en mención. Estos datos muestran entonces unas intenciones por superar las barreras puestas a las personas, pero, al mismo tiempo, una serie de contradicciones que vale la pena analizar. Es precisamente en este contexto donde comienza la búsqueda de mecanismos como la educación, que conllevan a materializar cuestiones como las garantías de acceso al conocimiento sin exclusión alguna (Hernández y Velásquez, 2016).

Al respecto, la legislación ha tenido unos desarrollos evidenciados en la Declaración Universal de los Derechos Humanos, la Declaración Mundial sobre Educación y el Decreto 366 del 9 de febrero de 2009, de acuerdo con Padilla (2012), en 
el contexto educativo habría una baja proporción de profesores con las competencias para acompañar el aprendizaje de personas con discapacidad. Para llegar a este tipo de afirmación, se realizó una pesquisa en ciertos contextos educativos mediante la utilización de un cuestionario que contenía 39 ítems en lo referente al tema de la discapacidad en estudiantes. Algunos de los puntos de análisis recogidos aquí, indican que los docentes dan una amplia diversidad de respuestas en relación con el conocimiento de los tipos de discapacidad, dando cuenta del conocimiento que ellos tienen al respecto y de las experiencias personales vinculadas a esta cuestión.

Además, según esta investigación, dicho resultado sería la evidencia de una baja preparación de los docentes para asumir el reto de la enseñanza en el contexto de la discapacidad, así como también se plantea que esto podría afectar notablemente los procesos de inclusión, en términos de facilitar un desarrollo de capacidades de los individuos. Por lo tanto, para promover la inclusión, debe comprenderse a las personas con discapacidad como sujetos de derechos y, en este sentido, pensar en la interacción con los docentes ubicando en el centro de la discusión pedagógica, el desarrollo de las personas con dificultades para acceder al conocimiento (Padilla, 2012).

Teniendo como referente este panorama, se encuentran varias iniciativas que en el fondo evidencian la transformación de una actitud tanto académica como política, para superar esta visión excluyente hacia las personas con $\mathrm{SD}$ en Colombia. En concordancia con esto, se encuentra el trabajo de Castañeda (2012), quien plantea una estrategia para facilitar la capacidad de comunicación entre los niños con SD y sus profesoras. Más en detalle, propone unos tipos de comunicación denominado aumentativa y alternativa, que define como un conglomerado de métodos, modos y estrategias de comunicación que son usados por las personas que posibilita el intercambio de contenidos con el entorno (Sánchez y Torres, 1997; citados por Castañeda,
2012). Este tipo de comunicación es, además, utilizado por las personas cuando tienen alguna imposibilidad de hacerlo de manera oral, por lo tanto, suministra un canal de interrelación hasta que pueda restablecerse la comunicación formal.

Las herramientas de apoyo para esta comunicación pasan por el habla, los gestos de uso común, signos manuales, recursos gráficos, etc. Concluye entonces que es necesario poner en discusión el aspecto comunicativo en la relación entre profesores y estudiantes con SD. Si bien, este estudio aporta solo un ejercicio llegando a unos resultados parciales, es importante destacar el nuevo lugar que las maestras dan a los estudiantes mediante un carácter reflexivo de su práctica docente, pues también genera una nueva sensibilidad frente al tema de la discapacidad cognitiva (Castañeda, 2012). Seguramente, al seguir estos análisis, los maestros van a abordar con nuevos ojos la problemática que enfrentan a diario al incluir, mediante diferentes herramientas pedagógicas, a sus aprendices.

En una vía similar al caso anterior, Flórez, Moreno, Bermúdez, y Cuervo (2009), exponen una propuesta realizada en el marco del proyecto llamado Educación Superior Inclusiva promovido por la Universidad Nacional de Colombia. En el documento donde se presenta la cuestión, se proponen unos lineamientos que pretenden recoger el discurso de la inclusión para llevarlo a un contexto práctico. Por ejemplo, plantea la necesidad de promover la formación de docentes para que lleven a cabo estrategias de flexibilidad curricular, de modo que estén en la capacidad de articularse a las necesidades de cada persona de acuerdo a su limitación. A partir de estas normativas, se articulan objetivos enfocados en generar y promover programas que faciliten la construcción del tejido social, creando una conciencia de la comunidad en general frente a los procesos de inclusión de estudiantes en situación de discapacidad. Tales estrategias deberían transmitirse a los docentes, directivos, personal 
administrativo y técnico implicado en el proceso educativo. Asimismo, esto debería ser tarea de estudiantes con y sin discapacidad, así como también padres de familia (Flórez et al., 2009).

En concordancia con esto, Ramírez (2004) propone que para romper las barreras de acceso al conocimiento, es necesario que las instituciones educativas generen diversos tipos de acercamiento en función de la didáctica, de modo que no estén elaboradas desde las limitantes que traen los estudiantes al llegar al espacio de aprendizaje. De igual modo, señala como importante la incorporación al currículo de los aportes de la psicología cognitiva y las tecnologías de información, facilitando a los estudiantes espacios de interacción social donde puedan desarrollar nuevas competencias tanto en la comunicación, como en su desarrollo intelectual (Ramírez, 2004).

Así las cosas, al pensar esta cuestión en Colombia, se encuentran un obstáculo relacionado con la educación y es que esta permanece segmentada enfatizando en las deficiencias de la persona. La causa de esto, estaría en la carencia de unos mecanismos que entrelacen programas y modelos teóricos que sean coherentes entre sí. Para trabajar en la resolución de esto, Ramírez (2004), propone establecer una integración entre los sistemas educativos regulares y los especializados, garantizando una igualdad en el proceso de desarrollo cognitivo y comunicativo de las personas con SD. De igual manera, la propuesta considera la incorporación de tecnologías de información como instrumentos de apoyo para promover la construcción de herramientas cognitivas en las personas, pensando en asuntos primordiales como el aprendizaje de la lecto-escritura. En tal sentido, las personas con SD podrían tener posibilidades reales de ampliar sus habilidades de comprensión lectora y escritora $y$, por tanto, se constituye en algo de vital importancia, dado que se promoverían el desarrollo de sus habilidades cognitivas y no se partiría desde sus limitantes (Ramírez, 2004).

\section{Conclusión}

En el desarrollo de la comprensión del SD queda expuesta una relación con las condiciones históricas, evidenciando así cambios que pretenden mostrar el abandono de unas perspectivas deterministas, por unas que ponen al individuo en centro del análisis como una posibilidad de ser. Los ejemplos de las denominaciones como débiles mentales o el tratamiento eugénesico (Kühl, 1994), responden a una preocupación por encontrar unas condiciones de normalidad y poder ajustar a los individuos a estos parámetros. Se encuentra, además, un énfasis por encontrar la discapacidad en el sustrato biológico del ser humano, es decir, el cuerpo es la fuente y el punto de llegada de la comprensión del fenómeno de la discapacidad, además que su entendimiento está fijado a la noción de enfermedad.

La búsqueda del equilibrio del cuerpo y, por tanto, la sanación a través de la verdad científica utilizando mecanismos de rehabilitación (Palacios y Romañach, 2008), permitieron pues el encuadramiento del cuerpo humano, el cual, una vez entrado en alguna limitación sea del orden físico o mental, formulan la necesidad de transformarlo y recuperarlo según el orden normal de la naturaleza. No obstante, siguiendo el señalamiento que hacen Palacios y Romañach (2008), al entrar en el modelo social, valores como la igualdad, libertad y dignidad cobran importancia permitiendo re evaluar la mirada normalizadora, además, accediendo a nuevas miradas que consideran al SD como una condición que, como otras, plantea necesidades distintas que las sociedades deben atender y formas diversas de ocupar un lugar en el entramado social

Los cambios al respecto quedan evidenciados en diferentes contextos. Para el caso del SD, fueron mencionados la educación en concordancia con la 
propuesta de una legislación que proporcionara un norte. Sin embargo, las leyes por sí solas no son garantía del cambio de percepción sobre esta condición, por tanto, es necesario implementar estrategias que faciliten una transformación en el pensamiento de todos los ciudadanos y comprendan que muchas limitaciones que determinan las condiciones de vida de las personas con SD, son construidas socialmente.

Los discursos jurídicos como la Declaración Mundial sobre Educación para Todos o la Convención de los Derechos de las Personas con Discapacidad (entre otros) pretendieron, pues, superar la brecha entre conocimiento científico y una perspectiva social de la discapacidad. La educación, por ende, constituye una vía para lograr disminuir, en la práctica, las brechas presentadas a las personas con $\mathrm{SD}$, puesto que es en la construcción de conocimientos significativos para la vida donde puede marcarse una diferencia. Por esta razón, la reflexión acerca del tema educativo debe orientarse en el reconocimiento de las potencialidades del individuo, dejando de lado las miradas deterministas de la visión enfermedad -normalización, propias del paradigma rehabilitador -médico sobre la discapacidad.

Si bien es necesario reconocer que este es un proceso largo, cambios a pequeña escala como la formación de docentes, conformación de grupos de reflexión constituidos por familias de este contexto y la articulación con espacios institucionales (Estado y sus dependencias), pueden contribuir a la transformación tanto de las propias personas en condición de SD y la sociedad en general. Por ejemplo, en el trabajo con las familias, puede contribuirse a través del rescate de las experiencias valiosas, una re significación de los procesos de crianza en tanto a la enfermedad se le otorga un sentido distinto y los vínculos familiares son fortalecidos (Collazos y Herrera, 2017).

\section{Referencias Bibliográficas}

Azevedo, L.M y Damasceno.L (2013). Marriage and reproduction in a woman with Down syndrome. International medical review on down'S syndrome. (17), 39-42.

Bell, L. (2017) Babies with Down Syndrome Deserve Love, Not Eradication. Pregnancy Help News. Recuperado de https://pregnancyhelpnews.com/babies-withdown-syndrome-deserve-love-not-eradication

Bowman-Kruhm, M. (2003). Everything You Need to Know about Down Syndrome. New York: The Rosen Publishing Group.

Brill, M. T. (2006). Down Syndrome. New York: Marshall Cavendish

BBC (2016). La prueba médica que podría acabar con el síndrome de Down. Recuperado de http://wwww.bbc. com/mundo/noticias-37524744

Carr, J. y Carr, J. H. (1995). Down's Syndrome: Children Growing Up. New York: Cambridge University Press

Castañeda, M. I. (2012). Estrategias para potenciar las habilidades comunicativas de las maestras del Jardín Nueva Esperanza de la localidad de Bosa y los niños con síndrome de Down a través de la comunicación Aumentativa y Alternativa (tesis inédita de especialización). Universidad Pedagógica Nacional, Bogotá, Colombia.

Córdoba, L; Henao, C. y Verdugo, M.A. (2016). Calidad de vida de adultos colombianos con Discapacidad Intelectual. Hacía la promoción en Salud. 21 (1), 91-105.

Córdoba, L; Mora, A; Bedoya, A; Verdugo, M.A. (2007) Familias de Adultos con Discapacidad Intelectual en Cali, Colombia, Desde el Modelo de Calidad de Vida. PSYKHE. 16 (2), 29 - 42.

Convención internacional sobre los derechos de las personas con discapacidad. (s.f.). Recuperado de http://www.un.org/spanish/disabilities/ convention/convention.html 
Collazos, T. y Herrera, E. (2017) Metas y estrategias parentales en padres de hijos con Síndrome de Down. Inédito. Fundación Universitaria Católica Lumen Gentium, Santiago de Cali.

Crosso, Camila. (2010) El derecho a la educación de personas con Discapacidad: Impulsando el concepto de Educación Inclusiva. Revista Latinoamericana de Educación Inclusiva. 4 (2), 79 - 95.

Departamento Administrativo Nacional de Estadística DANE (año). Censo General 2005. Recuperado de https://www.dane.gov.co/index.php/estadisticas-por-tema/demografia-y-poblacion/censo-general-2005-1

Diagnostic Criteria for Intellectual Disabilities: DSM-5 Criteria - Intellectual Disabilities. (s.f). Recuperado de http://www.gulfbend.org/poc/view_doc.php?type $=$ doc $\&$ id $=10348 \& \mathrm{cn}=208$

Esteba-Castillo, S; Ribas, N; Baró, D \& Novell, R. (2006). Healthy ageing in people with Down syndrome and dementia: the need to foster education and support programs for individuals, families and organizations. International Medical Journal On Down Syndrome. (10),, 25-29.

Flórez, R; Moreno, M; Bermúdez, G. y Cuervo, G. (2009). Lineamientos de política para la atención educativa de poblaciones en situación de discapacidad en las instituciones de educación superior en Colombia. Areté. 9, 11-24

Garvía, B y Ruf, P. (2014). Living as a couple with Down's syndrome. International Medical Review on Down's Syndrome. 18, (1), 9-12.

Gómez, V. (2005). La anomia para las personas con discapacidad (tesis inédita de licenciatura. Universidad de las Américas Puebla, Puebla, México.

González, E. (2010). Medicina y Nacismo. Aprender de la historia. Elservier Doima. 211,4. Recuperado de https://www.clinicalkey.es/\#!/content/ playContent/1-s2.0-S0014256511000543?returnurl=http:\%2F\%2Flinkinghub.elsevier.com \% 2Fretrieve\%2Fpii\%2FS0014256511000543\%3Fshowall $\% 3$ Dtrue \& referrer $=$ https: $\% 2 F \% 2 F s c h o l a r$. google.es $\% 2 F$
Hernández, A. (2017) Experiencia Internacional en Derechos de las Personas con Discapacidad. La Silla Rota. Recuperado dehttp://lasillarota.com/opinion/ columnas/experiencia-internacional-en-derechos-de-las- personas-con-discapacidad/138877

Hernández, E. y Velásquez, J. (2016). Comprensiones del discurso normativo sobre inclusión educativa en Colombia. Ágora, 16 (2), 493-512.

Hernández - Jaramillo, Janeth, Hernández - Uman, 7, Iván (2005). una aproximación a los costos indirectos a la discapacidad en Colombia. Revista de salud pública, 7 (2), 130-144. Retraer agosto 23, 2019. from: www.scielo.org.co/scielo.php?script=sciottextpie $\mathrm{d}=50124$ _00642500020002esing=es

Hurtado Lozano, L., \& Agudelo Martínez, M. (2014). Inclusión educativa de las personas con discapacidad en Colombia. (Educational inclusion for the disabled in Colombia). CES Movimiento y Salud, 2(1), 45-55. Recuperado de http://revistas.ces.edu.co/index. php/movimientoysalud/article/view/2971

Inzúa, V. (México, 2001). Una conciencia histórica y la discapacidad. Revista trabajo social Nueva Época. 3. 76-83.

Keel, F. (2016). Down Syndrome: Health Issues. Kindle Edition.

Kûhl, S. (1994) The Nazi Connection. Eugenics, American Racism And German National Socialism. New York: Oxford University Press.

Lorenz, S. (1998) Children with Down's Syndrome: A guide for teachers and support assistants in mainstream primary and secondary schools. New York: David Fulton Publishers.

Maldonado, J. (2013) El modelo social de la discapacidad. Una cuestión de Derechos Humanos. Boletín Mexicano de Derecho Comparado. 138, 1093-1109.

Ministerio de Salud (año). Plan Nacional de Discapacidad 2005. Recuperado de https://www.minsalud.gov.co/

Nazer J.; Cifuentes O. (2011). Estudio epidemiológico global del síndrome de Down. Revista chilena de pediatría, 82(2), 105-112. Recuperado de https://dx.doi. org/10.4067/S0370-41062011000200004 
Noack, T., \& Fangerau, H. (2007). Eugenics, euthanasia, and aftermath. International Journal of Mental Health, 36(1), 112-124.

Organización de Naciones Unidas. (1956) El derecho a una vida normal: ayuda internacional en la rehabilitación de las personas con defectos físicos. Revista de las Naciones Unidas. 284, 46

Organización de Naciones Unidas (2016). Convención sobre los derechos de las personas con discapacidad. Comité sobre los Derechos de las Personas con Discapacidad. Observaciones finales sobre el informe inicial de Colombia. Recuperado de http://www.discapacidadcolombia.com/index.php/ colombia-se-raja-en-informe-presentado-a-la-onu

Padilla, A. (2012). Inclusión educativa de personas con discapacidad. Revista Colombiana de Psiquiatría. 40 (4), 670.

Palacios, A. y Romañach, J. (2008) El modelo de diversidad: una nueva visión de la bioética desde la perspectiva de las personas con diversidad funcional (discapacidad). Intersticios. Revista Sociológica de Pensamiento Crítico. 2 (2), 37-47.
Ramírez, D.A. (2004). Implicaciones didácticas de un estudio sobre habilidades relacionadas con la comprensión lectora en adolescentes con Síndrome de Down. Lectura y Vida, volumen (número), 11-24.

Reynolds, T; Zupanick, C.E y Dombeck, M. (s.f). What Is an Intellectual Disability? Recuperado de http://www.gulfbend.org/poc/view_doc. php?type $=$ doc\&id $=10324$

Valls Gubern, T. (2010). School stage and students with Down syndrome. International Medical Review On Down Syndrome. 14 (2), 31-33.

World Conference on Education for All(s.f.). Declaración Mundial sobre Educación para Todos y Marco de referencia para la acción encaminada a lograr la satisfacción de las necesidades básicas de aprendizaje. Recuperado de http:// unesdoc.unesco.org/images/0008/000862/086289sb. pdf

Yeepay, W., \& Chwen-, Y. S. (2012). Patterns of participation and enjoyment in adolescents with Down syndrome. Research in Developmental Disabilities. 33, 841-848. 